\title{
BMJ Open Racial differences of heart failure with midrange ejection fraction (HFmrEF): a large urban centre-based retrospective cohort study in the USA
}

\author{
Dan Leslie Li, ${ }^{\oplus 1}$ Renato Quispe, ${ }^{1,2}$ Chioma Onyekwelu, ${ }^{1}$ Robert T Faillace, ${ }^{3}$ \\ Cynthia C Taub ${ }^{4}$
}

To cite: Li DL, Quispe R, Onyekwelu C, et al. Racial differences of heart failure with midrange ejection fraction (HFmrEF): a large urban centrebased retrospective cohort study in the USA. BMJ Open 2019;9:e026479. doi:10.1136/ bmjopen-2018-026479

\section{- Prepublication history for} this paper is available online. To view these files please visit the journal online (http://dx.doi org/10.1136/bmjopen-2018026479).

Received 9 September 2018 Revised 19 December 2018 Accepted 11 February 2019

Check for updates

(C) Author(s) (or their employer(s)) 2019. Re-use permitted under CC BY-NC. No commercial re-use. See rights and permissions. Published by BMJ.

1 Internal Medicine, Jacobi Medical Center, Albert Einstein College of Medicine, Bronx, New York, USA

${ }^{2}$ Ciccarone Center for the Prevention of Heart Disease, The Johns Hopkins Schoold of Medicine, Baltimore, Maryland, USA

${ }^{3}$ Cardiology, Jacobi Medical Center, Albert Einstein College of Medicine, Bronx, New York, USA ${ }^{4}$ Cardiology, Montefiore Medical Center, Albert Einstein College of Medicine, Bronx, New York, USA

Correspondence to

Dr Cynthia C Taub;

ctaub@montefiore.org

\section{ABSTRACT}

Objectives We aimed to study the racial differences in clinical presentations, survival outcomes and outcome predictors among patients with heart failure (HF) with midrange ejection fraction (HFmrEF, EF 40\%-49\%).

Design This is a retrospective study.

Setting Adults with HF diagnosis at Montefiore Medical Center, Bronx, New York between 2008 and 2012, with an inpatient echocardiogram showing left ventricular ejection fraction of $40 \%-49 \%$ were included as HFmrEF population.

Participants $1,852 \mathrm{HFmrEF}$ patients are included in the study (56\% male, mean age 67 years). There were 493 (26.5\%) non-Hispanic whites, 541 (29.2\%) non-Hispanic black, 489 (26.4\%) Hispanics and 329 (17.8\%) other racial populations.

Outcome measures Cumulative probabilities of all-cause mortality among different racial groups were estimated and multivariable adjusted Cox proportional regressions were performed to assess predictors of mortality.

Results Among the HFmrEF patients, white patients were older and were less likely to be on guideline-directed medications. Blacks had a lower prevalence of prior myocardial infarction comparing to other groups. Hispanics had more chronic diseases and yet better survival comparing to whites and blacks after adjustment for age, sex and comorbidities. Distinct sets of survival predictors were revealed in individual racial groups. Baseline use of mineralocorticoid receptor antagonist (MRA) was associated with lower mortality among HFmrEF patients in general (HR 0.61, 95\% $\mathrm{Cl} 0.37$ to 0.99).

Conclusions There are significant racial/ethnic differences in clinical phenotypes, survival outcomes and mortality predictors of HFmrEF. Furthermore, the use of MRA predicted a reduced mortality in HFmrEF patients.

\section{INTRODUCTION}

The left ventricular ejection fraction (LVEF) has been a useful tool to clinically characterise subsets of heart failure (HF). Not only the LVEF value is independently associated with mortality, ${ }^{1}{ }^{2}$ but classification of HF defined by LVEF values distinguishes the pathophysiology of different HF phenotypes, ${ }^{3}$ and
Strengths and limitations of this study

- A large cohort of heart failure with midrange ejection fraction population equally representing blacks, Hispanics and whites was studied.

- Clinical characteristics and survival outcome were compared among different racial/ethnic groups.

- Predictors for mortality within each of the three race-ethnicity groups were demonstrated.

- The inclusion of heart failure patients was solely based on International Classification of Diseases, Ninth Revision codes.

- Volume matrix measurements were not readily incorporated as routine protocols at earlier time points, which contributed to some loss of echocardiographic data.

predicts responses to medical therapies. ${ }^{45}$ In 2016 European Society of Cardiology redefined the classification of HF by ejection fraction (EF) including a new category with EF of $40 \%-49 \%$ named HF with midrange ejection fraction (HFmrEF). ${ }^{6}$ Previously labelled as $\mathrm{HF}$ with reduced $\mathrm{EF}$ (HFrEF) or HF with preserved EF (HFpEF), HFmrEF is a grey zone of $\mathrm{HF}$ that needs better characterisation.

Studies on HFmrEF have started to emerge ${ }^{7}$; however, data remain scant, especially in racial-ethnically divergent populations. Most studies were done in white-predominant populations $^{8-10}$ and in some Asian populations. ${ }^{11} 12$ Nonetheless, to our knowledge, no studies exist that representatively include blacks and Hispanics in the USA.

In this hospital-based retrospective cohort study, we aim to examine clinical characteristics and survival outcomes of HFmrEF, in a racial/ethnically divergent community consisting largely of non-Hispanic white, non-Hispanic black and Hispanic individuals. 


\section{METHODS}

\section{Study population}

We included adult patients (older than 18 years of age) hospitalised in Montefiore Medical Center, Bronx, New York from 1 January 2008 to 31 December 2012, with a primary discharge diagnosis of HF (by International Classification of Diseases, Ninth Revision [ICD-9] codes) and an echocardiography performed during hospitalisation. For patients who had multiple admissions during that period, the first admission was selected as the index hospitalisation. We further excluded patients who deceased during the index hospitalisation. Patients with HFmrEF were further defined as LVEF in between $40 \%$ and $49 \%$ on the echocardiography performed during index hospitalisation. LVEF was assessed via biplane Simpson's method. The study was carried out after the approval from Institutional Review Board of Albert Einstein College of Medicine. We used the Strengthening of the Reporting of the Observational Studies in Epideomiology (STROBE) cohort study checklist when writing our report. ${ }^{13}$

\section{Data collection}

Clinical information was collected from electronic medical record using Clinical Looking Glass V.3.3 (CLG) - a patented software that collates medical records for research purpose. Basic clinical characteristics including age, sex, self-reported race/ethnicity, comorbidities defined by ICD-9 codes, and medications at the time of inclusion were collected for the study. Socioeconomic status (SES) was assessed using a summary Z-score that combined wealth, income and education levels, and reflected the deviation from the average SES in the New York state population (negative number-below; positive number-above). Echocardiographic data were also collected via CLG. The end of follow-up for this study was 30 June 2016. All-cause mortality was determined by either social security administration records or house staff notes.

\section{Statistical analysis}

Baseline characteristics were shown using descriptive statistics. Continuous variables were presented as mean (SD) for normally distributed data, or median (25th75th percentile) otherwise. Categorical variables were expressed as number of subjects and percentages. Baseline continuous variables were compared among groups using one-way analysis of variance test. Pearson's $\chi^{2}$ test was used for comparison of categorical/nominal variables. Cumulative probabilities of all-cause mortality were estimated using the Kaplan-Meier method. Multivariable Cox proportional hazards regression models were used to study the associating factors for all-cause mortality. We tested the proportional hazard assumptions graphically (using log-log plots and comparing KaplanMeier observed and Cox predicted curves), as well as numerically (using Schoenfeld residuals). A two-sided p-value $\leq 0.05$ was considered statistically significant. Analyses were performed using Stata V.13 (StataCorp).

\section{Patient and public involvement}

This study is based on a deidentified patient database. Patient and public involvement was not specifically sought in the investigation.

\section{RESULTS}

\section{Clinical features of heart failure with midrange ejection} fraction in a multiracial cohort

The study population consisted of 1852 patients, including 493 non-Hispanic whites $(26.6 \%), 541$ non-Hispanic blacks (29.2\%), 487 Hispanics (26.3\%) and 331 individuals $(17.9 \%)$ of other racial components (Asians, Pacific Islanders, American Indians and mixed race). Distinct clinical characteristics among different racial/ethnic groups were revealed (table 1). Non-Hispanic whites were older, had significantly higher prevalence of atrial fibrillation and lower prevalence of hypertension, diabetes and chronic kidney disease (CKD). Non-Hispanic blacks were featured by higher female percentage $(53.5 \%)$ in contrast to other two groups $(37.3 \%$ in whites and $41.3 \%$ in Hispanics), and with significantly lower prevalence of prior myocardial infarction (MI) (whites vs blacks vs Hispanics: $39.8 \%$ vs $27.4 \%$ vs $40.3 \%, \mathrm{p}<0.001$ ); Hispanics had the highest prevalence of diabetes (whites vs blacks vs Hispanics: $32.3 \%$ vs $41.2 \%$ vs $53.2 \%$, p<0.001) (table 1 ). The use of guideline-directed HF medications in general was lowest in non-Hispanic whites despite their better SES (table 1). The level of N-terminal pro b-type natriuretifc peptide (NT-proBNP) was the highest in non-Hispanic whites and the lowest in non-Hispanic blacks. Echocardiographic features were largely similar among the three race-ethnicity groups, except that Hispanics had lower average pulmonary artery systolic pressure (table 1).

\section{Survival outcome of heart failure with midrange ejection fraction in a multiracial cohort}

After a median follow-up time of 54 months, individuals with HFmrEF had a cumulative mortality of $36.5 \%$, with an incidence rate of 7 per 1000 person-months. Racial/ ethnic differences in mortality were observed (figure 1). We further adjusted the mortality with age and sex. In comparison to non-Hispanic whites, Hispanics had significantly reduced all-cause mortality (HR $0.74,95 \%$ CI 0.59 to 0.92 ), whereas no difference was observed between non-Hispanic blacks and whites (HR 0.89, 95\% CI 0.72 to 1.10). Even after adjustments for age, sex and clinical characteristics, the overall mortality of Hispanics remained significantly lower than the other two racial groups (HR $0.68,95 \%$ CI 0.54 to 0.86 , table 2 ).

Multivariable adjusted Cox regression analysis was performed to examine the predicting factors of all-cause mortality among patients with HFmrEF. Proportional hazard assumption was confirmed. Variables independently associated with all-cause mortality were outlined in table 2. Older age and CKD were associated with increased mortality in HFmrEF population, whereas previous MI was associated with a lower mortality in 
Table 1 Comparison of HFmrEF baseline clinical characteristics among racial/ethnic groups

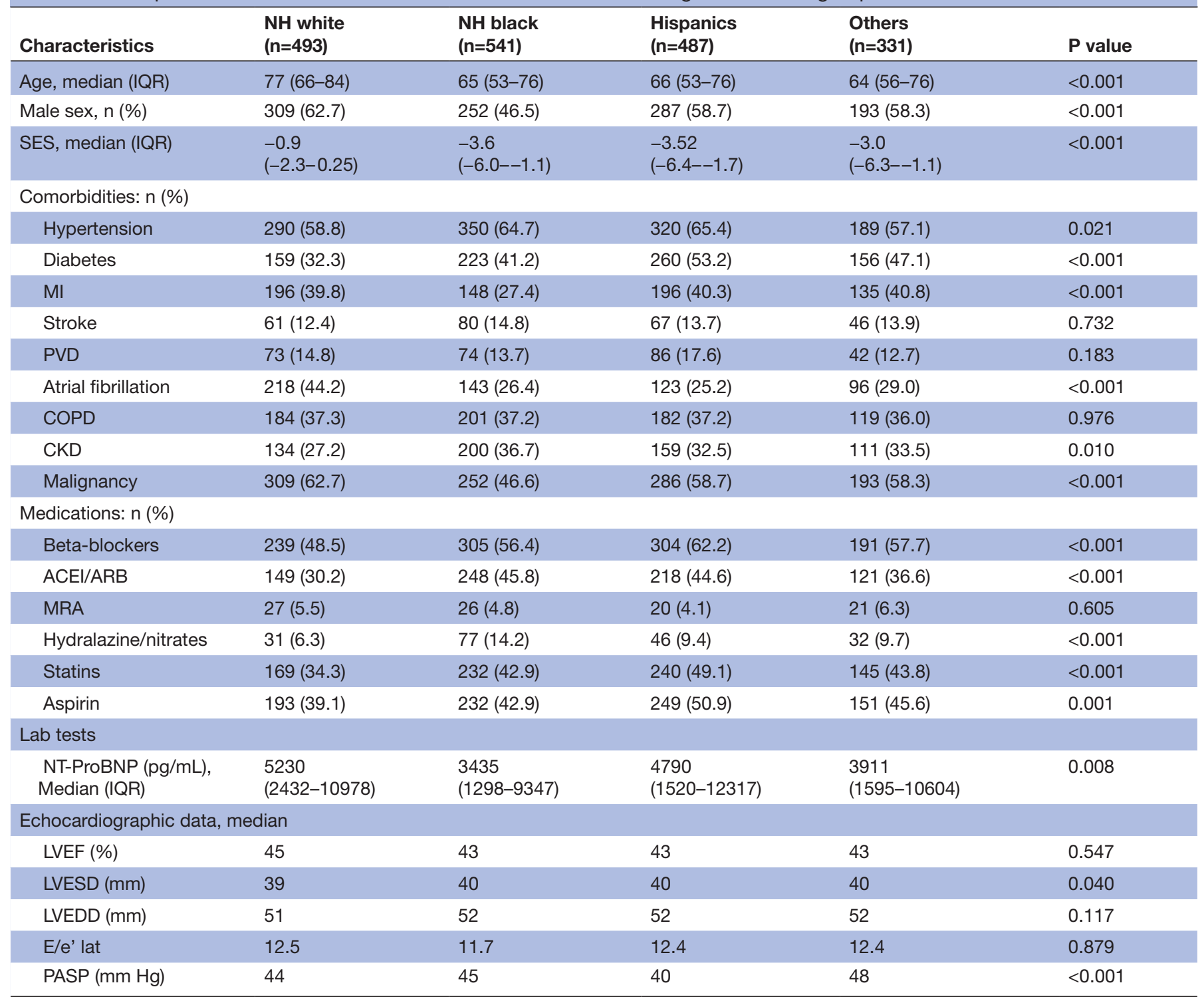

ACEI/ARB, Angiotensinogen-converting enzyme inhibitor/Angiotensin-receptor blocker; CKD, chronic kidney disease; COPD, chronic obstructive pulmonary disease; e' lat, lateral wall e'; HFmrEF, heart failure with midrange ejection fraction; LVEDD, left ventricular enddiastolic dimension; LVEF, left ventricular ejection fraction; LVESD, left ventricular end-systolic dimension; MI, myocardial infarction; MRA, mineralocorticoid receptor antagonist; NH black, non-Hispanic black; Others, including Asians, Pacific Islanders, American Indians and mixed-race; PASP, pulmonary artery systolic pressure. NH white, non-Hispanic white; PVD, peripheral vascular disease; SES, socioeconomic score.

HFmrEF (table 2). A higher baseline EF and baseline use of mineralocorticoid receptor antagonist (MRA) were associated with a lower mortality risk among HFmrEF patients. In comparison, baseline uses of other guideline-directed medical therapies including beta-blockers and angiotensin-converting enzyme inhibitors/angiotensin receptor blockers (ACEI/ARB) were not associated with a reduced mortality in HFmrEF (table 2).

To determine if predicting powers of any variables vary with race/ethnicity, we performed interaction testing with race/ethnicity and found three variables (peripheral vascular disease $[\mathrm{PVD}]$, atrial fibrillation, aspirin use) had effect modification ( $p<0.05$, table 3 marked in
‘*’). We further presented predicting factors in HFmrEF patients within each race-ethnicity group (table 3). PVD portended a significant risk of death only in non-Hispanic blacks (HR 1.933, 95\% CI 1.293 to 2.890), however failed to predict death in non-Hispanic whites and Hispanic cohorts; AF was significantly associated with increased death in non-Hispanic blacks (HR 1.449, 95\% CI 1.032 to 2.036) and Hispanics (HR 1.453, 95\% CI 1.001 to 2.109) but not in non-Hispanic whites (table 3). Potential roles of HF medications in predicting mortality in each racial/ ethnic group were largely inconclusive, except for aspirin in non-Hispanic blacks, likely due to the limitation of sample sizes (table 3). 


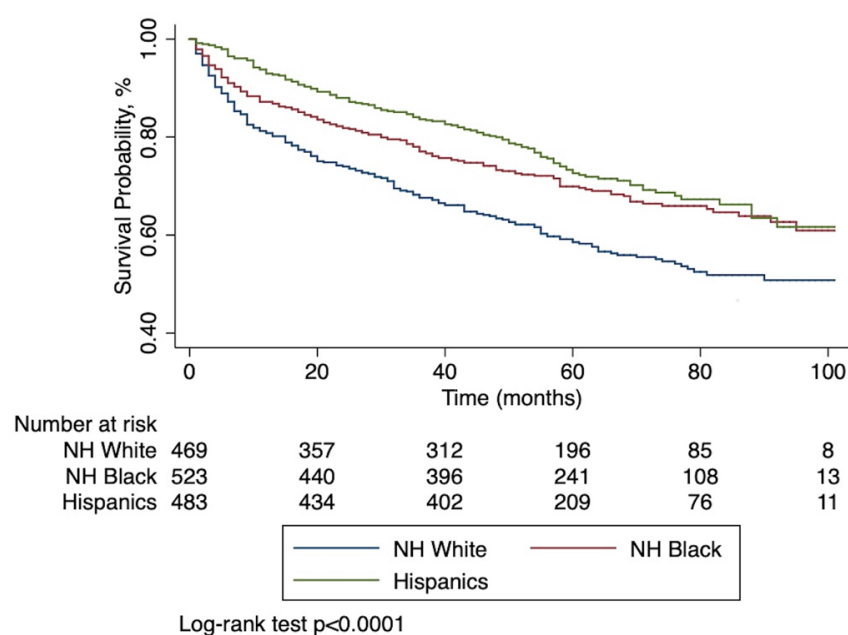

Figure 1 Overall survival in heart failure with midrange ejection fraction patients of different racial groups. $\mathrm{NH}$, nonHispanic.

\begin{tabular}{|c|c|}
\hline Covariates & HR (95\% Cl) \\
\hline Age, per year increase & 1.03 (1.02 to 1.04$)$ \\
\hline Sex (ref=female) & 1.01 (0.85 to 1.19$)$ \\
\hline Hypertension & 0.98 (0.82 to 1.17$)$ \\
\hline \multicolumn{2}{|l|}{ Race (ref=NH white) } \\
\hline NH black & 0.81 (0.65 to 1.01$)$ \\
\hline Hispanic & 0.68 (0.54 to 0.86$)$ \\
\hline Others & 0.96 (0.75 to 1.23$)$ \\
\hline Diabetes & 1.15 (0.97 to 1.35$)$ \\
\hline Ml & 0.80 (0.67 to 0.95$)$ \\
\hline Stroke & 1.07 (0.86 to 1.34$)$ \\
\hline PVD & 1.22 (0.98 to 1.51$)$ \\
\hline COPD & 1.08 (0.92 to 1.28$)$ \\
\hline CKD & 1.87 (1.57 to 2.23 ) \\
\hline Malignancy & 1.22 (0.92 to 1.61$)$ \\
\hline $\mathrm{AF}$ & 1.18 (0.99 to 1.40$)$ \\
\hline$E F$, per \% increase & 0.95 (0.92 to 0.98 ) \\
\hline Beta-blockers & 0.94 (0.77 to 1.16$)$ \\
\hline ACEI/ARB & 0.96 (0.80 to 1.17$)$ \\
\hline MRA & 0.61 (0.37 to 0.99$)$ \\
\hline Statins & 0.83 (0.67 to 1.03$)$ \\
\hline ASA & 0.83 (0.66 to 1.03 ) \\
\hline
\end{tabular}

Numbers in bold and italic: statistically significant for predicting mortality

ACEI/ARB, Angiotensinogen-converting enzyme inhibitor/ Angiotensin-receptor blocker; $A F$, atrial fibrillation; ASA, aspirin; CKD, chronic kidney disease; COPD, chronic obstructive pulmonary disease; EF, Ejection Fraction; HFmrEF, heart failure with midrange ejection fraction; MI, myocardial infarction; MRA, mineralocorticoid receptor antagonist; $\mathrm{NH}$ black, non-Hispanic black; NH white, non-Hispanic white; Others, including Asians, Pacific Islanders, American Indians and mixed-race. PVD, peripheral vascular disease.

\section{DISCUSSION}

Despite a few recent studies on HFmrEF since the new classification of $\mathrm{HF}$ according to $\mathrm{EF},{ }^{7}$ this new $\mathrm{HF}$ group is still not well characterised in racially and ethnically divergent populations. Here we present a comprehensive study on HFmrEF in a racially divergent community equally representing blacks, Hispanics and whites. We identified the racial differences of clinical features among patients with HFmrEF. Further, our data showed a reduced risk of mortality among Hispanics compared with white individuals, while no difference of mortality was observed in between non-Hispanic black and white individuals. Distinctive panels of predicting factors for death were also revealed within each race-ethnicity group. Additionally, we showed that baseline use of MRA was associated with reduced mortality, whereas use of beta-blockers and ACEI/ARB had no associated benefits among patients with HFmrEF.

Distinct clinical characteristics existed among different racial/ethnic groups of HFmrEF cohort. In general, Non-Hispanic whites were almost 10 years older than the two other racial/ethnic groups, however with lower prevalence of comorbidities including hypertension, diabetes and CKD. The proportion of patients with atrial fibrillation was highest in non-Hispanic whites with HFmrEF. Consistently, the prevalence of atrial fibrillation is known to be higher in Non-Hispanic whites comparing to Non-Hispanic blacks, Asians and Hispanics. ${ }^{14} 15$ The reason of the difference is unclear, although some reported the association of larger left atrium in Caucasian population with the higher atrial fibrillation prevalence. $^{15}$ Interestingly, they were less likely to be on medications proven to improve survival in HFrEF. It's not clear whether it was because they had higher rate of intolerance, contraindications to the medications, or due to other reasons. Blacks are known to have higher prevalence of coronary heart disease ${ }^{16}$; however in our study, Non-Hispanic blacks with HFmrEF had the lowest proportion of individuals with prior MI, suggesting that other factors-perhaps hypertension and CKD-had substantial contribution in the development of HFmrEF in this population. NT-Pro-BNP level was lowest in black individuals in comparison to whites and Hispanics. In line with this data, pooled resulted from multiple big community study registries showed that plasma NT-pro-BNP levels in blacks are significantly lower in as compared with whites, ${ }^{17}$ likely due to genetic variations.

We further demonstrated distinctive predictors of mortality among HFmrEF patients in general as well as in individual racial groups. In contrast to a recent study showing ischaemic aetiology of $\mathrm{HF}$ as a predictor of mortality in HFmrEF patients, ${ }^{18}$ our data showed that prior MI was negatively associated with mortality in HFmrEF. Rastogi et $a l^{19}$ showed that HFmrEF patients whose EF improved from prior-the population also with a higher prevalence of $\mathrm{CAD}$ - had significantly better mortality outcome than other HFmrEF patients. It is likely that in our population, prior MI history distinguishes a 
Table 3 Predictors of HFmrEF mortality by racial groups

\begin{tabular}{|c|c|c|c|c|}
\hline Covariates & $\begin{array}{l}\text { NH white } \\
\text { HR }(95 \% \mathrm{CI})\end{array}$ & $\begin{array}{l}\text { NH black } \\
\text { HR }(95 \% \mathrm{CI})\end{array}$ & $\begin{array}{l}\text { Hispanic } \\
\text { HR }(95 \% \mathrm{Cl})\end{array}$ & $\begin{array}{l}\text { Others } \\
\text { HR }(95 \% \mathrm{Cl})\end{array}$ \\
\hline Age, per year & $\begin{array}{l}1.04 \\
(1.03 \text { to } 1.06)\end{array}$ & $\begin{array}{l}1.02 \\
\text { (1.01 to } 1.03)\end{array}$ & $\begin{array}{l}1.01 \\
\text { (1.00 to } 1.03)\end{array}$ & $\begin{array}{l}1.05 \\
(1.04 \text { to } 1.06)\end{array}$ \\
\hline MI & $\begin{array}{l}0.79 \\
(0.58 \text { to } 1.08)\end{array}$ & $\begin{array}{l}0.80 \\
(0.55 \text { to } 1.15)\end{array}$ & $\begin{array}{l}0.94 \\
(0.65 \text { to } 1.36)\end{array}$ & $\begin{array}{l}0.66 \\
(0.43 \text { to } 1.01)\end{array}$ \\
\hline PVD* $^{*}$ & $\begin{array}{l}1.03 \\
(0.70 \text { to } 1.50)\end{array}$ & $\begin{array}{l}1.93 \\
\text { (1.29 to } 2.89)\end{array}$ & $\begin{array}{l}1.24 \\
(0.87 \text { to } 1.78)\end{array}$ & $\begin{array}{l}1.15 \\
(0.64 \text { to } 2.06)\end{array}$ \\
\hline CKD & $\begin{array}{l}1.96 \\
(1.45 \text { to } 2.66)\end{array}$ & $\begin{array}{l}1.95 \\
\text { (1.40 to } 2.71)\end{array}$ & $\begin{array}{l}1.79 \\
(1.24 \text { to } 2.60)\end{array}$ & $\begin{array}{l}1.73 \\
(1.05 \text { to } 2.84)\end{array}$ \\
\hline $\mathrm{AF}^{*}$ & $\begin{array}{l}0.96 \\
(0.72 \text { to } 1.50)\end{array}$ & $\begin{array}{l}1.50 \\
(1.03 \text { to } 2.04)\end{array}$ & $\begin{array}{l}1.45 \\
(1.00 \text { to } 2.11)\end{array}$ & $\begin{array}{l}1.12 \\
(0.70 \text { to } 1.77)\end{array}$ \\
\hline Beta-blockers & $\begin{array}{l}0.82 \\
(0.58 \text { to } 1.18)\end{array}$ & $\begin{array}{l}0.82 \\
(0.54 \text { to } 1.23)\end{array}$ & $\begin{array}{l}1.36 \\
(0.88 \text { to } 2.10)\end{array}$ & $\begin{array}{l}1.05 \\
(0.60 \text { to } 1.83)\end{array}$ \\
\hline MRA & $\begin{array}{l}0.68 \\
(0.30 \text { to } 1.58)\end{array}$ & $\begin{array}{l}0.81 \\
(0.32 \text { to } 2.04)\end{array}$ & $\begin{array}{l}0.59 \\
(0.18 \text { to } 1.88)\end{array}$ & $\begin{array}{l}0.53 \\
(0.16 \text { to } 1.75)\end{array}$ \\
\hline Statins & $\begin{array}{l}0.93 \\
(0.64 \text { to } 1.36)\end{array}$ & $\begin{array}{l}0.95 \\
(0.62 \text { to } 1.45)\end{array}$ & $\begin{array}{l}0.73 \\
(0.47 \text { to } 1.15)\end{array}$ & $\begin{array}{l}0.65 \\
(0.36 \text { to } 1.16)\end{array}$ \\
\hline $\mathrm{ASA}^{*}$ & $\begin{array}{l}1.09 \\
(0.75 \text { to } 1.59)\end{array}$ & $\begin{array}{l}0.66 \\
(0.45 \text { to } 0.99)\end{array}$ & $\begin{array}{l}0.96 \\
(0.61 \text { to } 1.51)\end{array}$ & $\begin{array}{l}0.67 \\
(0.38 \text { to } 1.18)\end{array}$ \\
\hline
\end{tabular}

Numbers in bold and italic: statistically significant for predicting mortality.

${ }^{*}$ P-interaction by race/ethnicity $<0.05$.

ACEI/ARB, Angiotensinogen-converting enzyme inhibitor/Angiotensin-receptor blocker; AF, atrial fibrillation; ASA, aspirin; Cl, Confidence Interval; CKD, chronic kidney disease; MI, myocardial infarction; MRA, mineralocorticoid receptor antagonist; NH black, non-Hispanic black; $\mathrm{NH}$ white, non-Hispanic white; Others, including Asians, Pacific Islanders, American Indians and mixed-race.; PVD, peripheral vascular disease

population of $\mathrm{HF}$ patients with improved $\mathrm{EF}$, who had better outcome among HFmrEF patients. The association between prior MI or diagnosis of CAD with mortality in patients with HF needs further study in the future. Distinctive from other recent studies, our study included diverse racial and ethnic groups. Genetic variations have been shown to contribute to the racial differences in neurohormonal signalling as well as the natural history and responses to medical therapies in $\mathrm{HF}^{20}$ An analysis including 47149 hospitalised HF individuals from Get With The Guideline-HF registry reported lower 1-year mortality in Hispanics and blacks in comparison with whites, even after adjustment for clinical and SES variables. ${ }^{21}$ No further comparisons among HF categories by EF were available. In our study, a similar mortality risk was found in blacks comparing to whites among HFmrEF population. On the other hand, Hispanic ethnic origin was an independent factor associated with a lower mortality despite their lower SES in comparison to non-Hispanic white individuals; we have further data suggesting that Hispanic patients had lower mortality risk (adjusted for covariates) compared with other racial groups in HFrEF and HFpEF population as well (data not shown). These observations perhaps echo previous epidemiological studies suggesting 'the Hispanic paradox'. ${ }^{22}$ Explanations of 'the Hispanic paradox' have been tempted and might be multitude, including dietary factors, social/family support and possibly genetic/epigenetic differences. ${ }^{22} 23$ Future studies are needed to confirm this finding, as well as to explore underlying mechanisms. Furthermore, our study presented distinctive predictors for mortality within each of the three race-ethnicity groups. While CKD was an equally strong predictor of mortality regardless of racial/ethnic groups, age, PVD and atrial fibrillation portended different mortality risks among non-Hispanic whites, non-Hispanic blacks and Hispanics. It is unknown whether this observation was merely due to differences of medical care accessibility, medications or interventional therapy (eg, PVD revascularisation strategies, anticoagulation therapy for $\mathrm{AF}$ and so on), versus genetic and metabolic variations. These observations need to be further examined in future studies; nonetheless, it highlights the differences among different race-ethnicity groups in HF population.

In contrast to HFrEF, no therapy thus far has proven to improve survival in HFpEF patients in clinical trials. As a recently defined entity and previously classified either as HFrEF and HFpEF in clinical trials, there is no evidencebased therapies recommended to improve survival in HFmrEF patients. After adjustment of covariates, we showed that only baseline use of MRA was associated with a lower mortality in HFmrEF. The benefit of MRA 
was shown as a reduction of re-hospitalisation in Treatment of Preserved Cardiac Function Heart Failure with an Aldosterone Antagonist study. In this study, a stronger benefit of MRA was seen in patients with LVEF $45 \%-50 \%$. No survival benefit of MRA was observed in the trial, however, many attributed the negative finding to the drastically different outcome results from Russia/Georgia in comparison to Americas, which probably resulted from inadequate actual use of MRA in the medication arm. ${ }^{24}{ }^{25}$ Therefore, MRA could be further studied as a potential survival-promoting medication for HFmrEF patients. Our study did not show a survival benefit of ACEI/ARB or beta-blocker use in HFmrEF population. The role of ACEI/ARB in HF patients with LVEF $>40 \%$ was variable in recent studies, ${ }^{8912}$ as well as in clinical trials. ${ }^{26} 27$ Beta-blockers were not found to significantly influence outcomes in patients with HFpEF as well as HFmrEF in an observational study using Organized Program to Initiate Lifesaving Treatment in Hospitalized Patients with Heart Failure registry. ${ }^{28}$ Therefore, further large population-based studies and clinical trials are needed to prove benefits of medications for HFmrEF patients.

Our study has several limitations. First, the inclusion of the HF patients in our study was based on ICD-9 of HF. There was no validation study to confirm the reliability of HF diagnosis. Second, our HFmrEF population might include a proportion of patients with HF with recovered/ improved EF, who might have different survival outcome than HF patients with initial EF 40\%-49\%. Future studies are needed to better categorise these two populations. Third, this is a retrospective study including patients from 2008 to 2012, volume matrix measurements were not readily incorporated as routine protocols at earlier time points, and contributed to some loss of echocardiographic data. Finally, the study is based on a single-centre patient population. Future studies from multiple institutions and areas are needed to confirm our findings.

\section{CONCLUSIONS}

In this large hospital-based cohort, we present contemporary data on clinical phenotypes of patients with HFmrEF in a multiracial/ethnic community. Our data uniquely showed racial/ethnically distinctive survival outcomes in individuals with HFmrEF. Distinct sets of mortality predictors among different racial/ethnic groups of HFmrEF patients might help clinical risk stratification. Use of MRA was associated with improved survival in patients with HFmrEF. Future studies are needed to further validate our findings.

Acknowledgements We would like to thank Nidhi Madan for advice on the conception of the study.

Contributors DLL and RQ contributed to the study's conception and design. DLL acquired the data. DLL, RQ, CO, RTF and CT contributed to the analysis and interpretation of the data. DLL and RQ drafted the manuscript. RTF and CT contributed to the critical revision.

Funding The authors have not declared a specific grant for this research from any funding agency in the public, commercial or not-for-profit sectors.
Competing interests None declared.

Patient consent for publication Not required.

Ethics approval The study protocol was proved by Institutional Review Board of Albert Einstein College of Medicine.

Provenance and peer review Not commissioned; externally peer reviewed.

Data sharing statement No additional unpublished data are available.

Open access This is an open access article distributed in accordance with the Creative Commons Attribution Non Commercial (CC BY-NC 4.0) license, which permits others to distribute, remix, adapt, build upon this work non-commercially, and license their derivative works on different terms, provided the original work is properly cited, appropriate credit is given, any changes made indicated, and the use is non-commercial. See: http://creativecommons.org/licenses/by-nc/4.0/.

\section{REFERENCES}

1. Cohn JN, Johnson GR, Shabetai R, et al. Ejection fraction, peak exercise oxygen consumption, cardiothoracic ratio, ventricular arrhythmias, and plasma norepinephrine as determinants of prognosis in heart failure. The V-HeFT VA Cooperative Studies Group. Circulation 1993;87:VI5-16.

2. Cintron G, Johnson G, Francis G, et al. Prognostic significance of serial changes in left ventricular ejection fraction in patients with congestive heart failure. The V-HeFT VA Cooperative Studies Group. Circulation 1993;87:VI17-23.

3. Borlaug BA, Redfield MM. Diastolic and systolic heart failure are distinct phenotypes within the heart failure spectrum. Circulation 2011;123:2006-14. discussion 2014.

4. McMurray JJ. Clinical practice. Systolic heart failure. N Engl J Med 2010;362:228-38.

5. Redfield MM. Heart Failure with Preserved Ejection Fraction. N Engl J Med 2016;375:1868-77.

6. Ponikowski P, Voors AA, Anker SD, et al. 2016 ESC Guidelines for the diagnosis and treatment of acute and chronic heart failure: The Task Force for the diagnosis and treatment of acute and chronic heart failure of the European Society of Cardiology (ESC). Developed with the special contribution of the Heart Failure Association (HFA) of the ESC. Eur J Heart Fail 2016;18:891-975.

7. Hsu JJ, Ziaeian B, Fonarow GC. Heart Failure With Mid-Range (Borderline) Ejection Fraction: Clinical Implications and Future Directions. JACC Heart Fail 2017;5:763-71.

8. Fonarow GC, Stough WG, Abraham WT, et al. Characteristics, treatments, and outcomes of patients with preserved systolic function hospitalized for heart failure: a report from the OPTIMIZE-HF Registry. J Am Coll Cardiol 2007;50:768-77.

9. Koh AS, Tay WT, Teng THK, et al. A comprehensive population-based characterization of heart failure with mid-range ejection fraction. Eur $J$ Heart Fail 2017;19:1624-34.

10. Cheng RK, Cox M, Neely ML, et al. Outcomes in patients with heart failure with preserved, borderline, and reduced ejection fraction in the Medicare population. Am Heart J 2014;168:721-30.

11. Lam CSP, Gamble GD, Ling LH, et al. Mortality associated with heart failure with preserved vs. reduced ejection fraction in a prospective international multi-ethnic cohort study. Eur Heart J 2018;39:17701780.

12. Tsuji K, Sakata Y, Nochioka K, et al. Characterization of heart failure patients with mid-range left ventricular ejection fraction-a report from the CHART-2 Study. Eur J Heart Fail 2017;19:1258-69.

13. von Elm E, Altman DG, Egger M, et al. The Strengthening the Reporting of Observational Studies in Epidemiology (STROBE) statement: guidelines for reporting observational studies. Lancet 2007;370:1453-7.

14. Dewland TA, Olgin JE, Vittinghoff $E$, et al. Incident atrial fibrillation among Asians, Hispanics, blacks, and whites. Circulation 2013;128:2470-7.

15. Marcus GM, Olgin JE, Whooley M, et al. Racial differences in atrial fibrillation prevalence and left atrial size. Am J Med 2010;123:375. e1-375.e7.

16. Saab KR, Kendrick J, Yracheta JM, et al. New insights on the risk for cardiovascular disease in African Americans: the role of added sugars. J Am Soc Nephrol 2015;26:247-57.

17. Bajaj NS, Gutiérrez OM, Arora G, et al. Racial Differences in Plasma Levels of N-Terminal Pro-B-Type Natriuretic Peptide and Outcomes: The Reasons for Geographic and Racial Differences in Stroke (REGARDS) Study. JAMA Cardiol 2018;3:11-17.

18. Chioncel O, Lainscak M, Seferovic PM, et al. Epidemiology and one-year outcomes in patients with chronic heart failure and 
preserved, mid-range and reduced ejection fraction: an analysis of the ESC Heart Failure Long-Term Registry. Eur J Heart Fail 2017;19:1574-85

19. Rastogi A, Novak E, Platts AE, et al. Epidemiology, pathophysiology and clinical outcomes for heart failure patients with a mid-range ejection fraction. Eur J Heart Fail 2017:19:1597-605

20. Taylor MR, Sun AY, Davis G, et al. Race, common genetic variation, and therapeutic response disparities in heart failure. JACC Heart Fail 2014;2:561-72.

21. Vivo RP, Krim SR, Liang L, et al. Short- and long-term rehospitalization and mortality for heart failure in 4 racial/ethnic populations. J Am Heart Assoc 2014;3:e001134.

22. Medina-Inojosa J, Jean N, Cortes-Bergoderi M, et al. The Hispanic paradox in cardiovascular disease and total mortality. Prog Cardiovasc Dis 2014;57:286-92.

23. Horvath S, Gurven M, Levine ME, et al. An epigenetic clock analysis of race/ethnicity, sex, and coronary heart disease. Genome Biol 2016;17:171.
24. de Denus S, O'Meara E, Desai AS, et al. Spironolactone Metabolites in TOPCAT - New Insights into Regional Variation. N Engl J Med 2017;376:1690-2.

25. Pfeffer MA, Claggett B, Assmann SF, et al. Regional variation in patients and outcomes in the Treatment of Preserved Cardiac Function Heart Failure With an Aldosterone Antagonist (TOPCAT) trial. Circulation 2015;131:34-42.

26. Massie BM, Carson PE, McMurray JJ, et al. Irbesartan in patients with heart failure and preserved ejection fraction. $N$ Engl $J$ Med 2008;359:2456-67.

27. Yusuf S, Pfeffer MA, Swedberg K, et al. Effects of candesartan in patients with chronic heart failure and preserved leftventricular ejection fraction: the CHARM-Preserved Trial. Lancet 2003;362:777-81.

28. Hernandez AF, Hammill BG, O'Connor CM, et al. Clinical effectiveness of beta-blockers in heart failure: findings from the OPTIMIZE-HF (Organized Program to Initiate Lifesaving Treatment in Hospitalized Patients with Heart Failure) Registry. J Am Coll Cardiol 2009;53:184-92. 